\title{
The Empty Notebook and Code Alert
}

The empty notebook has nothing to say about the murders in Rwanda, the massacres in Hebron and Haifa. The empty notebook insists its slate is clean. It is changing its cover to orange then red and back to yellow. Terrified by rumors, it listens to bulletins on the hour, receives intermittent briefings from unreliable sources. How can it tell where danger lies - in the street, in the air, on the shelf? The empty notebook interrogates a random sample of all who cross its path. It avoids racial profiling, is responsible to no one. The empty notebook has an empty conscience. 